\title{
The Effect of Cholinesterase Activity on the Diagnosis and Prognosis of Sepsis
}

\author{
Oznur Koylu ${ }^{1, *}$, Mehmet Yortanli ${ }^{2}$ \\ ${ }^{1}$ Konya Training and Research Hospital, Biochemistry Department, Konya, Turkey \\ ${ }^{2}$ Konya Training and Research Hospital, Emergency Department, Konya, Turkey \\ Email address: \\ drkoylu@mynet.com (O. Koylu) \\ ${ }^{*}$ Corresponding author
}

To cite this article:

Oznur Koylu, Mehmet Yortanli. The Effect of Cholinesterase Activity on the Diagnosis and Prognosis of Sepsis. Clinical Medicine Research. Vol. 5, No. 3, 2016, pp. 28-34. doi: 10.11648/j.cmr.20160503.13

Received: March 31, 2016; Accepted: April 14, 2016; Published: April 28, 2016

\begin{abstract}
Background: There are many biomarkers defined for systemic inflammation and sepsis. Cholinesterase and its biological role is not entirely known but in recent studies, it was seen that cholinesterase levels had a diagnostic value in predicting both sepsis and mortality. Objective: The purpose of this study is to establish the role of cholinesterase activity as a biomarker in the early diagnosis and treatment planning of sepsis which is an uncontrolled inflammatory response of the host to an infection. Materials and Method: This is a controlled, observational, and prospective clinical study and has been carried out on patients admitted to the intensive care unit with sepsis. The demographic features, the medical history and vital findings of the patients were recorded. According to the intensive care monitoring and treatment procedures, the complete blood count test, urine test and routine biochemical assessments particularly the CRP, procalcitonin and blood gasses tests were performed and the serum cholinesterase activity was assessed. The data was digitalized and then analyzed using the SPSS 15.0 software package. Results: The cholinesterase levels detected in the patient group were lower than the cholinesterase levels of the control group and there was a significant difference between the groups $(\mathrm{p}<0.001)$. A statistically significant association was detected between the severity of sepsis and the cholinesterase levels of the patients. There was also a statistical relationship between the cholinesterase levels and being connected to mechanical ventilation and the use of vasopressors $(\mathrm{p}<0.05)$. There was a significant association between mortality and cholinesterase levels $(p=0.009)$. As the cholinesterase activity decreased the mortality rate increased. As a result of the ROC analyses performed to establish the diagnostic value of the patients' cholinesterase levels in predicting sepsis and morality it was seen that cholinesterase levels had a diagnostic value in predicting both sepsis and mortality. Conclusions: We believe that the cholinesterase activity investigated in our study is an extremely useful biomarker in the diagnosis and prognosis prediction of the sepsis syndrome that progresses with systemic inflammation.
\end{abstract}

Keywords: Sepsis, Cholinesterase, Inflammation

\section{Background}

Inflammation is a response given by the organism to endogenous or various microorganisms, foreign bodies or toxins, it is necessary for the continuation of life, but it is non-specific. The biological purpose of this response is to repair the cellular injury [1]. Whatever the external stimulant, the response given to these stimulants are the same [2]. Systemic inflammation is a complex and dynamic process and is generally associated with poor outcomes [3]. Review of the literature will show that there are many biomarkers defined for systemic inflammation and sepsis such as procalcitonin, WBC (white blood cell), c-reactive protein etc [4]. It is not well-known before but we think that one of these biomarkers is the cholinesterase activity. In this study we aimed to emphasize the importance of cholinesterase activity on the diagnosis and prognosis of sepsis.

Cholinergic neural conduction plays an important role in the regulation of immune response during inflammation [5]. Increased vagal stimulation during inflammation inhibits the peripheral cytokine release through the nicotinic acetylcholine receptors [6]. 
Sepsis is defined as the uncontrolled inflammatory response of the host to infection. If sepsis that begins with the systemic response of the body to infection cannot be taken under control it may cause organ failure or death $[7,8]$. In the last 20 years the incidence of sepsis has increased 20 times, according to the data of the United States of America (USA) 700000 new cases of sepsis are diagnosed every year and sepsis is responsible for the death of 200000 patients annually [9]. Sepsis is of major importance as it is the most serious cause of death in non-coronary intensive care units [10]. The regulation of the release of mediators and cytokines that take part in the pathophysiology of sepsis is critically important for normal homeostasis and body resistance. On the other hand, the excessive or deficient release of these mediators leads to the damage of some organs, multiple organ failure and death $[11,12]$. Many cytokines take part in the development of SIRS and sepsis. The most important of these are IL-1, and TNF alpha and beta $[13,14]$.

There are two types of cholinesterases. True cholinesterase or cholinesterase I is found in the erythrocytes, lung, spleen, nerve endings and brain. It hydrolyses the neurotransmitter acetylcholine released from the nerve endings at synapses and facilitates the depolarization of the nerve. Pseudocholinesterase or cholinesterase II is found in the liver, pancreas, white matter of the brain and serum. Therefore, it is used as a liver function test. It is a marker of liver functions. Its biological role is not entirely known. The serum levels of the enzyme are used in clinical practice. Its normal range is very wide. It changes between 5400-13200 $\mathrm{U} / \mathrm{L}$ in men and 4300-11500 U/L in women.

\section{Objectives}

The purpose of this study is to establish the role and contribution of the identification of cholinesterase activity in the early diagnosis and treatment planning of sepsis that progresses with systemic inflammation and that is very fatal when treatment is delayed.

\section{Materials and Methods}

This study is a controlled, observational, and prospective clinical study and was conducted in the intensive care unit of the Emergency Department of the Konya Education and Research Hospital. Written consent was obtained from all patients or their legal guardians and all of the healthy volunteers. This study has been carried out within the framework of the Helsinki Declaration accepted by the World Medical Association (WMA) that defines the ethical principles of medical research carried out on human volunteers.

Fifty-eight patients over the age of 18 that had suspicion of diffuse systemic inflammation and indications for intensive care unit admission according to the international sepsis guidelines [15]. and 39 healthy adult volunteers were included in the study.

The demographic features, the medical history and vital findings of the patients were recorded. According to the intensive care monitoring and treatment procedures, appropriate samples for the complete blood count test, urine test and routine biochemical assessments particularly the CRP, procalcitonin and blood gasses tests were obtained. MULTIGENT Cholinesterase kits in ARCHITECT Systems were used to perform the kinetic colorimetric detection of cholinesterase.

Diagnostic radiological imaging studies and advanced tests to identify the causative microorganism were ordered. In addition, it was recorded whether the patients required mechanical ventilation and whether they received vasopressors. The duration of stay in intensive care and the mortality rate were also recorded. The APACHE II and SOFA scoring systems were used for prognostic evaluation. The control group consisted of healthy volunteers with no apparent comorbid disease and no sign of infection over the age of 18 .

Standard and early goal-directed treatment protocols were applied according to the international sepsis guidelines in the management of the patient group. Until the causative microorganism was isolated, empirical antibiotics were delivered and it was switched to the appropriate antibiotic according to the results of the culture antibiogram.

Statistical Analysis

The data was digitalized and then analyzed in the SPSS 15.0 software package. Visual (histogram and probability graphics) and analytic methods (KolmogorovSmirnov/Shapiro-Wilk tests) were used to determine if the variables were distributed normally. The numerical data obtained from the descriptive analyses of the normally distributed data are given as mean \pm standard deviation, nonnormal data are given as interquartile range (IQR) and nominal data are given as percentage. $\mathrm{P}$ values $<0.05$ were accepted as statistically significant in the statistical analyses performed.

The age values were normally distributed in the statistical analysis and the patient group and the control group were compared using the Student $t$ test. The patient group was compared with the control group with respect to gender using the Chi-square test. As the cholinesterase levels displayed nonnormal distribution, the use of mechanical ventilation and vasopressors, and mortality rates of the patient group and the control group were analyzed using the Mann-Whitney U test, and the Kruskal-Wallis test was used to analyze the diagnosis and severity of sepsis. The Receiver Operating Characteristics (ROC) curve was used to examine the decision-making characteristics of the cholinesterase levels in predicting mortality and sepsis. The sensitivity, specificity, positive predictive value and negative predictive value were calculated for the significant threshold values. In the evaluation of the area under the curve, conditions in which the Type-1 error rate is under 5\% were interpreted as conditions in which the diagnostic value of the test is statistically significant.

\section{Results}

Our study was conducted as a prospective study in the Emergency Department of the Konya Research and Training 
Hospital between July 2015 and February 2016 and included patients that were diagnosed with sepsis in the emergency department that were admitted to the intensive care unit. In total, 97 patients were included in the study, 58 comprised the case group and 39 comprised the control group.

The mean age of the patient group was $71,3 \pm 16,27$, and the mean age of the control group was $36,95 \pm 17,47$ and there was a statistically significant difference $(p<0.01)$. The patient group had $30(51.7 \%)$ male and $28(48.3 \%)$ female cases. The control group had $15(38.5 \%)$ male and 24 $(61.5 \%)$ female cases. There was no difference between the groups with respect to gender $(\mathrm{p}=0.109)$. The cholinesterase level was $3707.1740 \mathrm{U} / \mathrm{L}(3155.1678)$ in the patient group and $9187.5380 \mathrm{U} / \mathrm{L}(958.5405)$ in the control group, and there was a significant difference between the groups $(p<0.001)$ (Figure 1).

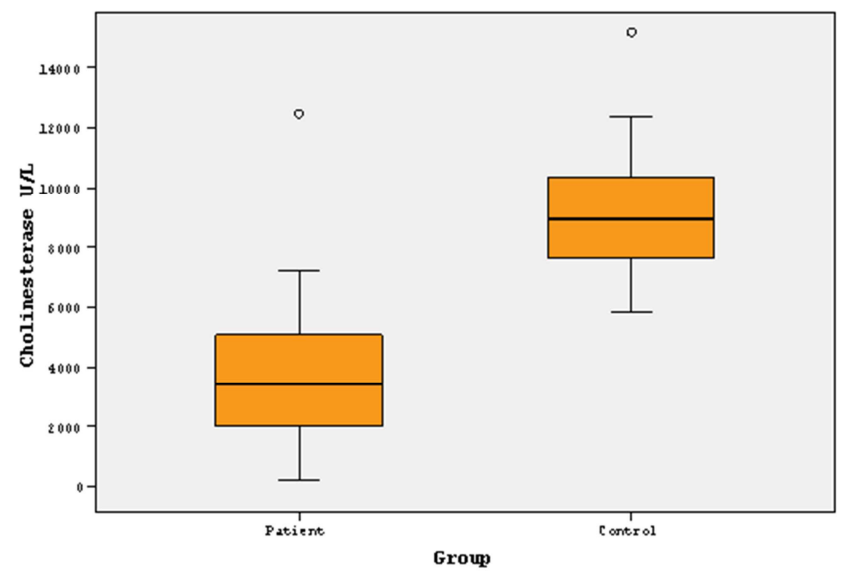

Figure 1. The box-point chart of cholinesterase levels.

The patients were divided into 4 groups according to the area of origin of sepsis;24 $(41.4 \%)$ cases were diagnosed with sepsis originating from the respiratory system, 22 $(37.9 \%)$ with sepsis from the urinary system, $6(10.3 \%)$ with sepsis from soft tissue infections and $6(10.3 \%)$ with sepsis from intraabdominal infections. No relationship was identified with respect to the cholinesterase levels according to the origin of sepsis ( $\mathrm{p}=0.837$ ).

The patients were divided into three groups according to the severity of sepsis as sepsis, severe sepsis and septic shock; $36(\% 62,1)$ patients were placed in the sepsis group, $13(\% 22,4)$ in the severe sepsis group, and $9(\% 15,5)$ in the septic shock group. A statistically significant association was detected between the severity of sepsis and the cholinesterase levels of the patients $(\mathrm{p}=0.003)$ (Table 1$)$.

Table 1. The comparative table of the severity of sepsis and the cholinesterase levels of the patients.

\begin{tabular}{llll}
\hline severity of sepsis & $\begin{array}{l}\text { Number, } \\
\mathbf{n}(\%)\end{array}$ & $\begin{array}{l}\text { cholinesterase, U/L, } \\
\text { median (IQR) }\end{array}$ & p value \\
\hline sepsis & $36(\% 62,1)$ & $4152,871(2572,3493)$ & \\
severe sepsis & $13(\% 22,4)$ & $2037,029(2230,2212)$ & 0,003 \\
septic shock & $9(\% 15,5)$ & $2340,968(2753,8468)$ & \\
\hline
\end{tabular}

From the history of the patients diagnosed with sepsis, 15 $(25.9 \%)$ had coronary artery disease, 22 (32.8\%) had hypertension, $14(24.1 \%)$ had cerebrovascular disease, 19 $(32.8 \%)$ had chronic obstructive pulmonary disease, 14 $(24.1 \%)$ had diabetes mellitus, $12(20.7 \%)$ had chronic kidney failure, and $3(5.2 \%)$ had a malignity. No association was detected between the patient histories and their cholinesterase levels ( $p>0.05)$.

The number of patients that required mechanical ventilation support was $13(22.4 \%)$ and the number of patients that were delivered vasopressors was 24 (41.4\%). A significant association was identified between the cholinesterase levels and being connected to a mechanical ventilator and the use of vasopressors $(\mathrm{p}<0.05)$ (Table 2$)$.

Table 2. The comparative table of being connected to mechanical ventilation and the use of vasopressors with cholinesterase levels.

\begin{tabular}{llllll}
\hline & & Number, n(\%) & $\begin{array}{l}\text { cholinesterase, U/L, } \\
\text { median (IQR) }\end{array}$ & p value \\
\hline $\begin{array}{l}\text { mechanical } \\
\text { ventilation }\end{array}$ & $(+)$ & $13(\% 22,4)$ & $2637,7260(2327,0417)$ & 0,023 \\
& $(-)$ & $45(\% 77,6)$ & $4016,1260(3359,8804)$ & \\
$\begin{array}{l}\text { use of } \\
\text { vasopressors }\end{array}$ & $(+)$ & $24(\% 41,4)$ & $2752,3650(2304,9021)$ & 0,003 \\
\hline
\end{tabular}

No growth was observed in the blood cultures of 28 (48.3\%) of the cases, Escherichia coli was detected in 9 (15.5\%), Staphylococcus spp. in 7 (12.1\%), Acinetobacter baumannii in 7 (12.1\%), Pseudomonas aeruginosa in 4 $(6.9 \%)$ and Enterococcus supp. in $3(5.2 \%)$ of the cases. No significant relationship was detected between the culture results and the cholinesterase levels $(\mathrm{p}=0.396)$.

Among the sepsis patients $28(48.3 \%)$ patients died. The cholinesterase level of in-hospital mortality patients was 2937.930 U/L (2329.0397) and the cholinesterase level of the patients with no in-hospital mortality was 4250.679 (3124.0479). There was a significant association between mortality and cholinesterase levels $(p=0.009)$

In the ROC analysis performed to investigate the diagnostic value of cholinesterase levels for predicting mortality, it was seen that cholinesterase levels have a diagnostic value for predicting mortality (AUC: 0,699, \%95GA: 0,56-0,83, $\mathrm{p}=0,009)$. The cut-off value for the cholinesterase results was determined as $\leq 4018.2834$, the sensitivity as $78.6 \%$, the specificity as $60 \%$, the positive predictive value $64.7 \%$ and the negative predictive value as $75 \%$ (Figure-2).In the ROC analysis performed to evaluate the diagnostic value of cholinesterase levels in predicting sepsis, it was seen that cholinesterase levels have a diagnostic value for predicting sepsis (AUC: 0,975, \%95GA: 0,94-1,01, $\mathrm{p}<0,001)$. The cut-off value of cholinesterase levels for predicting sepsis was determined as $\leq 6976,8198 \mathrm{U} / \mathrm{L}$, the sensitivity as $96.6 \%$, the specificity as $94.9 \%$, the positive predictive value as $96.6 \%$ and the negative predictive value as $94.9 \%$ (Figure 3 ). 


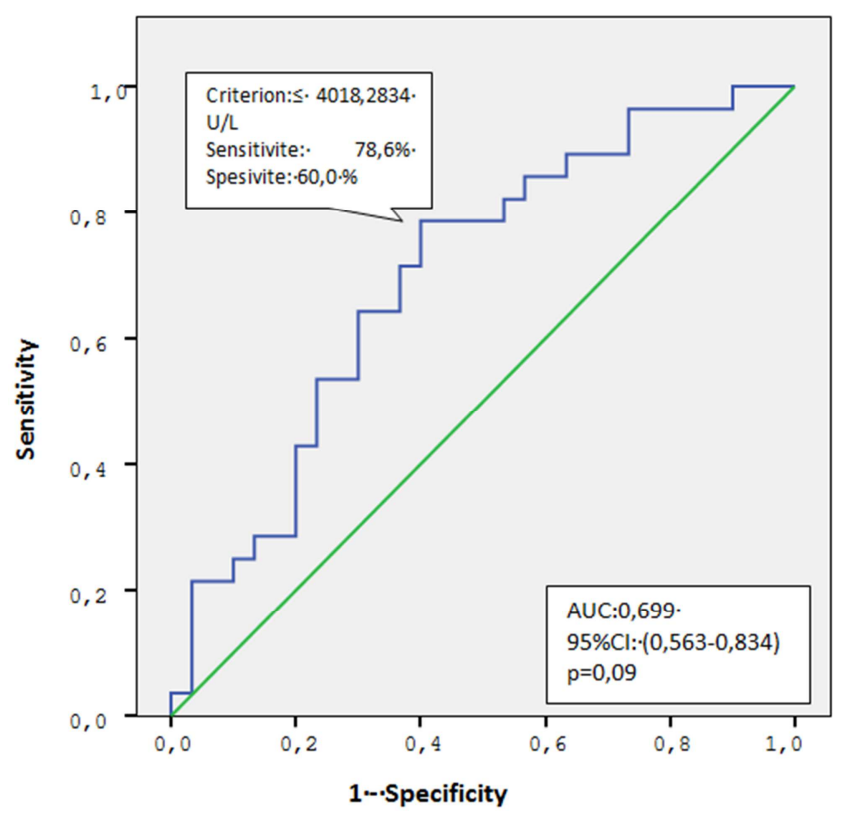

Figure 2. The ROC analysis of cholinesterase levels in the prediction of mortality.

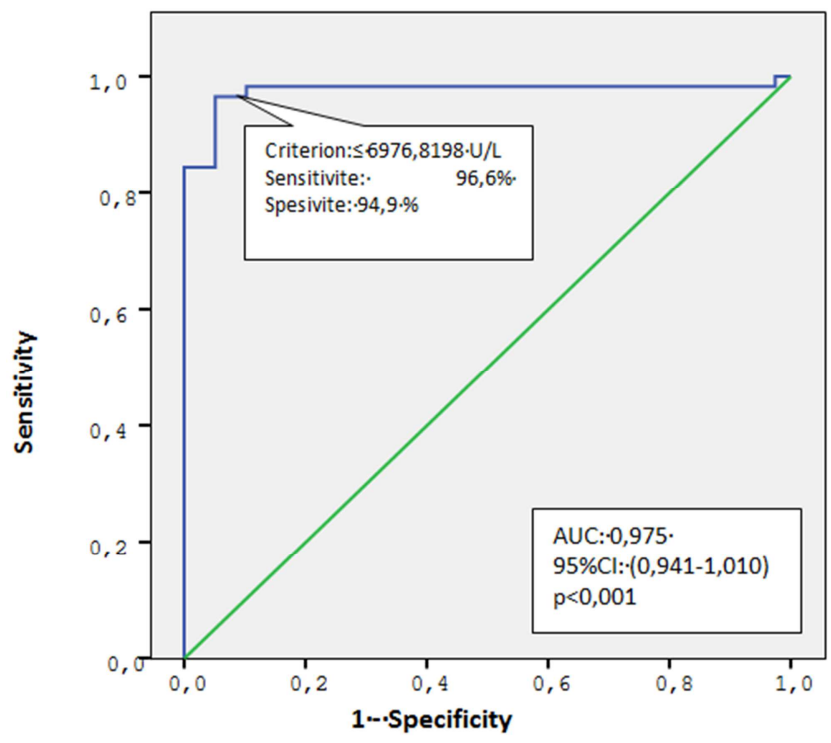

Figure 3. The ROC analysis of cholinesterase levels in predicting sepsis.

\section{Discussion}

The cholinesterase activity of the patient group that was admitted to intensive care with sepsis [3707.1740 U/L (3155.1678)] is significantly lower than the cholinesterase activity of the control group [9187.5380 U/L (2958.5405)] consisting of healthy individuals with no findings of inflammation and this difference is statistically very significant $(\mathrm{p}<0.001)$. Additionally, when the cholinesterase levels are evaluated according to the severity of sepsis, it is seen that as the clinical condition worsens the cholinesterase activity decreases. The fact that this condition has statistical significance $(p=0.003)$ indicates that cholinesterase activity may be accepted as an important novel biomarker that can be useful during diagnosis of systemic diseases with high mortality rates, particularly sepsis and in predicting their prognosis. As much as it is important to improve the understanding of pathophysiology to diagnose and treat sepsis, it is also important to equip physicians with knowledge about the early diagnosis and treatment of sepsis. It has been shown that increased probability of survival is especially associated with the knowledge of the intensive care physicians [16]. In a wide-scope study conducted in 7 state hospitals in the United States of America by Angus and colleagues, they determined that the number of patients with severe sepsis increased with age and that more than half of the patients are over 65 years of age [17]. In one prospective study conducted by Nguyen and colleagues the mean patient age was identified as 66 , and $63 \%$ of the patients were male and $37 \%$ were female [18].

In our study the mean patient age was $71,3 \pm 16,27$ and $51.7 \%$ were male and $48.3 \%$ were female. The mean age of the patients that died was $73,8 \pm 16,187$. Unlike literature studies, we only included patients diagnosed with sepsis that were admitted to the intensive care unit, we believe this has caused our mean age to be slightly higher. In the study conducted by Lai et al. in a tertiary hospital that included 262 patients that applied to the emergency department of the hospital, the most common bacterial infection in patients with sepsis was pneumonia $(n=82,40.2 \%)$ and this was followed by urinary system infections $(n=48,23.5 \%)$ [19].

In consistence with literature, the most common primary focus of infection that caused sepsis was pneumonia $(n=24$, $41.4 \%)$ followed by urinary system infections $(n=22,37.9 \%)$. However, when the patients with sepsis were classified according to the source of the infection, no significant difference was identified with respect to their cholinesterase levels $(\mathrm{p}=0.837)$.

The rate of microbiologically documented infections ranges between $59 \%$ and $68 \%$ in studies [20]. Blood cultures are one of the diagnostic criteria used for sepsis, however, it loses its value due to its low sensitivity (25-42\%) [21].

We also did not detect any growth in the blood culture samples of $28(48.3 \%)$ cases of our study. The microorganisms identified in the cases in which growth was detected were: Escherichia coli in 9 (15.5\%), Staphylococcus supp. in 7 (12.1\%), Acinetobacter baumannii in 7 (12.1\%), Pseudomonas aeruginosa in $4(6.9 \%)$ and Enterococcus supp. in $3(5.2 \%)$ of the cases. However, no significant association was identified between the culture results and the cholinesterase levels ( $\mathrm{p}=0.396)$.

In both of the studies conducted by Hines and colleagues [22], and Valles and colleagues [23], particularly gramnegative bacteria such as Escherichia coli and grampositive bacteria such as Staphylococcus, Streptococcus emerge as the most common causative microorganisms. With respect to causative organisms, our study also yielded similar results.

In our study, during the investigation of cholinesterase levels in the prognosis of sepsis, severe sepsis and septic shock, CRP, procalcitonin, WBC and lactate that are often used and are accepted as acute phase reactants were used as 
comparable parameters. An important disadvantage of CRP is the fact that its levels rise not only in bacterial infections but also in other inflammations and conditions of tissue destruction. In addition, its long half-life limits its guidance in monitoring and predicting the prognosis of dynamic clinical conditions like sepsis. Ugarte and colleagues monitored the daily CRP and procalcitonin levels of 205 patients being treated in the intensive care unit. They compared the values obtained during bacteremia, sepsis or when septic shock developed. It was discovered that the diagnostic sensitivity and specificity of procalcitonin was lower than the diagnostic sensitivity and specificity of CRP. It was also shown that the procalcitonin levels of the survivors were significantly higher. No similar association was demonstrated for CRP. It was stated that procalcitonin is a valuable parameter as a determinant of prognosis [24]. Suprin and colleagues reported significantly higher procalcitonin and CRP levels in septic shock patients than in patients with severe sepsis and SIRS [25]. Muller and colleagues, have demonstrated that there is a significant correlation between the severity of sepsis and the serum procalcitonin and CRP levels [26]. But in contrary, there are also studies that report that the CRP levels of patients with septic shock are lower than the CRP levels of patients with sepsis and severe sepsis and that CRP levels cannot be used as a marker that determines the severity of the disease [27]. In light of these studies, we attempted to demonstrate that cholinesterase levels can be a useful biomarker for determining the severity of the disease and also for determining the prognosis and mortality rate. Among the sepsis patients included in our study, $28(48.3 \%)$ patients died. The cholinesterase levels were significantly lower in the patients that died than in the survivors $(p=0.009)$.

As a result of the ROC analyses performed to establish the diagnostic value of the patients' cholinesterase levels in predicting sepsis and morality it was seen that cholinesterase levels had a diagnostic value in predicting both sepsis and mortality.

In a recent study conducted by Fenk and colleagues, it was identified that the cholinesterase activity was much higher in patients diagnosed with sepsis that responded to treatment and survived than it was in patients that died. The APACHE II scores of these patients were also low [28]. This condition is very consistent with the results of our study.

Setoguchi and colleagues induced sepsis by perforating the caecum in rats in their experimental study. They used the parasympatholytic agent distigmine bromide (a peripheral non-specific cholinesterase inhibitor) to control the production of cytokines that play an important role in the pathogenesis of sepsis and they determined that it suppressed the induction of inflammatory cytokines [29]. Chiarla and colleagues identified a significant decrease in the cholinesterase activity of patients in a critical condition, in particular, patients with sepsis or liver dysfunction [30]. Fernandez-Cabezudo and colleagues have shown that cholinergic stimulation of the immune system has a protective effect against salmonella infections [31].
In one other study including 26 patients, they expressed that reduced cholinesterase activity can be accepted as a specific marker of hepatic dysfunction and systemic sepsis syndrome in patients admitted to intensive care with the diagnosis of septic shock [32]. Our study includes a much higher number of cases $(n=58)$. We believe that the identification of cholinesterase activity will be very useful in conditions such as sepsis with a severe clinical course firstly in making the diagnosis and risk-scoring, and later in the treatment planning and prognosis prediction. Thus Wolkmer and colleagues have shown that pre-treatment with curcumin modulates acetylcholinesterase activity and proinflammatory cytokines in rats infected with Trypanosomaevansi [33]. The fact that we detected factors that negatively impacted prognosis such as increased mortality, increased requirement for mechanical ventilation, increased use of vasopressors, increased in-hospital stay in patients with reduced cholinesterase activity makes it necessary to perform more extensive studies on this biomarker.

\section{Conclusion}

After evaluating the findings we obtained in our study, we believe that the identification of cholinesterase levels will be extremely useful in the early diagnosis, prognosis prediction, treatment planning and even when making the call to transfer the patient to a better equipped medical center earlier, particularly in patients with clinical conditions that progress with systemic inflammation. As stated in every treatment guide, early treatment saves lives. Key points we obtained from our study: The cholinesterase levels of the patient group were lower than the control group and there was a significant difference between the groups $(\mathrm{p}<0.001)$. There was a statistically significant association between the severity of sepsis and the cholinesterase levels of the patients $(p=0.003)$. A statistically significant association was shown between the cholinesterase levels and being connected to a mechanical ventilator and the use of vasopressors $(p<0.05)$. There was a significant association between mortality and cholinesterase levels $(\mathrm{p}=0.009)$. As the cholinesterase activity decreased, an increase in mortality rates was observed.

As a result of the ROC analyses performed to establish the diagnostic value of the patients' cholinesterase levels in predicting sepsis and morality it was seen that cholinesterase levels had a diagnostic value in predicting both sepsis and mortality.

It is clear that laboratory analyses are necessary for the diagnosis, treatment planning and prognosis prediction of diseases. This becomes even more important in units such as intensive care units that treat critical patients. We believe that cholinesterase activity investigated in our study is a very useful biomarker for the diagnosis and prognosis prediction of the sepsis syndrome that progresses with systemic inflammation. However, these statements need to be supported by more extensive studies. 


\section{Limitations}

The small number of cases in the patient and control groups and the single-center nature of our study may be counted among the limitations of our study. We believe that the results will be more reliable when obtained from multicentric studies with larger groups that include various disease groups. One other limitation was the younger mean age of the control group $(36,95 \pm 17,47)$ when compared to the patient group $(71,3 \pm 16,27)$. However, it should be noted that it was difficult to find healthy volunteers that will give blood samples in the same age group as the patient group, and this was a limiting factor in our study.

\section{Acknowledgments}

We thank to intensive care unit staff for their help and support.

\section{References}

[1] Snyderman R, Gallin JI, Goldstein IM. Inflammation: basic principles and clinical correlates: Raven Press; 1992.

[2] Kumar V, Abbas AK, Fausto N, Aster J. Pathologic basis of disease. Philadelphia, PA: Elsevier Saunders; 2005.

[3] Alberti C, Brun-Buisson C, Goodman SV, Guidici D, Granton $\mathrm{J}$, Moreno $\mathrm{R}$, et al. Influence of systemic inflammatory response syndrome and sepsis on outcome of critically ill infected patients. American journal of respiratory and critical care medicine. 2003; 168 (1): 77-84.

[4] Pierrakos C, Vincent J-L. Sepsis biomarkers: a review. Crit Care. 2010; 14 (1): R15.

[5] Borovikova LV, Ivanova S, Zhang M, Yang H, Botchkina GI, Watkins LR, et al. Vagus nerve stimulation attenuates the systemic inflammatory response to endotoxin. Nature. 2000; 405 (6785): 458-62.

[6] Wang H, Yu M, Ochani M, Amella CA, Tanovic M, Susarla S, et al. Nicotinic acetylcholine receptor $\alpha 7$ subunit is an essential regulator of inflammation. Nature. 2003; 421 (6921): 384-8.

[7] Bone RC, Balk RA, Cerra FB, Dellinger RP, Fein AM, Knaus WA, et al. Definitions for sepsis and organ failure and guidelines for the use of innovative therapies in sepsis. The ACCP/SCCM Consensus Conference Committee. American College of Chest Physicians/Society of Critical Care Medicine. Chest Journal. 1992; 101 (6): 1644-55.

[8] Hotchkiss RS, Karl IE. The pathophysiology and treatment of sepsis. New England Journal of Medicine. 2003; 348 (2): 13850 .

[9] Fauci AS. Harrison's principles of internal medicine: McGraw-Hill Medical New York; 2008.

[10] Moss M, Martin G. A global perspective on the epidemiology of sepsis. Intensive care medicine. 2004; 30 (4): 527-9.

[11] Young E, Mandell G, Bennett J, Dolin R. Principles and practice of infectious diseases. Principles and Practice of Infectious Diseases. 2000.
[12] Lehr HA, Bittinger F, Kirkpatrick CJ. Microcirculatory dysfunction in sepsis: a pathogenetic basis for therapy? The Journal of pathology. 2000; 190 (3): 373-86.

[13] van der Poll T, van Deventer SJ. Cytokines and anticytokines in the pathogenesis of sepsis. Infectious disease clinics of North America. 1999; 13 (2): 413-26.

[14] Paterson RL, NR W. Sepsis and the SIRS: JR Coll Surg Edin 2000. $178-82 \mathrm{p}$

[15] Dellinger RP, Levy MM, Rhodes A, Annane D, Gerlach H, Opal SM, et al. Surviving Sepsis Campaign: international guidelines for management of severe sepsis and septic shock, 2012. Intensive care medicine. 2013; 39 (2): 165-228.

[16] Carson SS, Stocking C, Podsadecki T, Christenson J, Pohlman A, MacRae S, et al. Effects of organizational change in the medical intensive care unit of a teaching hospital: a comparison of open and closed formats. Jama. 1996; 276 (4): 322-8.

[17] Angus DC, Linde-Zwirble WT, Lidicker J, Clermont G, Carcillo J, Pinsky MR. Epidemiology of severe sepsis in the United States: analysis of incidence, outcome, and associated costs of care. Critical Care Medicine-Baltimore-. 2001; 29 (7): 1303-10.

[18] Nguyen DN, Spapen H, Su F, Schiettecatte J, Shi L, HachimiIdrissi S, et al. Elevated serum levels of S-100 $\beta$ protein and neuron-specific enolase are associated with brain injury in patients with severe sepsis and septic shock*. Critical care medicine. 2006; 34 (7): 1967-74.

[19] Lai CC, Chen SY, Wang CY, Wang JY, Su CP, Liao CH, et al. Diagnostic value of procalcitonin for bacterial infection in elderly patients in the emergency department. Journal of the American Geriatrics Society. 2010; 58 (3): 518-22.

[20] Boussekey N, Cantrel J, Dorchin Debrabant L, Langlois J, Devos P, Meybeck A, et al. Epidemiology, prognosis, and evolution of management of septic shock in a French intensive care unit: a five years survey. Critical care research and practice. $2010 ; 2010$.

[21] Edmond MB, Wallace SE, McClish DK, Pfaller MA, Jones RN, Wenzel RP. Nosocomial bloodstream infections in United States hospitals: a three-year analysis. Clinical infectious diseases. 1999; 29 (2): 239-44.

[22] DW H, JM L. Sepsis. Philadelphia: W. B. Saunders Company; 1998.

[23] Valles J, Rello J, Ochagavia A, Garnacho J, Alcalá MA. Community-acquired bloodstream infection in critically ill adult patients: impact of shock and inappropriate antibiotic therapy on survival. CHEST Journal. 2003; 123 (5): 1615-24.

[24] Povoa P, Coelho L, Almeida E, Fernandes A, Mealha R, Moreira $\mathrm{P}$, et al. $\mathrm{C}$ - reactive protein as a marker of infection in critically ill patients. Clinical microbiology and infection. 2005; 11 (2): 101-8.

[25] Suprin E, Camus C, Gacouin A, Le Tulzo Y, Lavoue S, Feuillu A, et al. Procalcitonin: a valuable indicator of infection in a medical ICU? Intensive care medicine. 2000; 26 (9): 1232-8.

[26] Müller B, Becker KL, Schächinger H, Rickenbacher PR, Huber PR, Zimmerli W, et al. Calcitonin precursors are reliable markers of sepsis in a medical intensive care unit. Critical care medicine. 2000; 28 (4): 977-83. 
[27] Brunkhorst F, Al-Nawas B, Krummenauer F, Forycki Z, Shah P. Procalcitonin, C-reactive protein and APACHE II score for risk evaluation in patients with severe pneumonia. Clinical microbiology and infection. 2002; 8 (2): 93-100.

[28] Feng W, Tang C, Guo H, Bao Y, Wen X, Xue T, et al. Prognostic value of serum cholinesterase activities in sepsis patients. Hepato-gastroenterology. 2012; 60 (125): 1001-5.

[29] Setoguchi D, Yatsuki H, Sadahiro T, Nakamura M, Hirayama Y, Watanabe E, et al. Effects of a peripheral cholinesterase inhibitor on cytokine production and autonomic nervous activity in a rat model of sepsis. Cytokine. 2012; 57 (2): 23844.

[30] Chiarla C, Giovannini I, Giuliante F, Vellone M, Ardito F, Nuzzo G. Plasma cholinesterase correlations in acute surgical and critical illness. Minerva chirurgica. 2011; 66 (4): 323-7.
[31] Fernandez - Cabezudo MJ, Lorke DE, Azimullah S, Mechkarska M, Hasan MY, Petroianu GA, et al. Cholinergic stimulation of the immune system protects against lethal infection by Salmonella enterica serovar Typhimurium. Immunology. 2010; 130 (3): 388-98.

[32] Al-Kassab A, Vijayakumar E. Profile of serum cholinesterase in systemic sepsis syndrome (septic shock) in intensive care unit patients. Clinical Chemistry and Laboratory Medicine. 1995; 33 (1): 11-4.

[33] Wolkmer P, da Silva CB, Paim FC, Duarte MM, Castro V, Palma HE, et al. Pre-treatment with curcumin modulates acetylcholinesterase activity and proinflammatory cytokines in rats infected with Trypanosoma evansi. Parasitology international. 2013; 62 (2): 144-9. 